\title{
Surveillance under improved supportive measures, as a safe approach to manage acute posttraumatic left renal ischemia. Case report
}

\begin{abstract}
Most traumatic kidney injuries are due to closed trauma caused by traffic or sports accidents. The validation of the ASST classification (American Society of the Surgery of Trauma) has led to a better analysis and management of those lesions. The occurrence of acute renal ischemia following trauma is a rare entity and is often associated with multi-organ dysfunction. There is no real recommendation for the management of this entity because of their low incidence, the delay in diagnosis and partly because of the diversity of the actors of the therapeutic decision. Our observation illustrates the safety of a conservative attitude in the management of a post-traumatic left renal ischemia, and through which we will resume the pathophysiological, diagnostic and therapeutic aspects.
\end{abstract}

Keywords: acute renal ischemia, sub intimal defect of renal artery, conservative attitude, renal injury
Volume 8 Issue 6 - 2018

\begin{abstract}
Adil Mellouki, Rhyan Alami Ouaddane, Mustapha Ahsaini, Soufiane Mellas, JD El Ammari, MF Tazi, MJ Fassi, Moulay Hassan Farih

Urology Department, University Hopital of Fès, Morocco
\end{abstract}

Correspondence: Adil Mellouki, Urology Department, University Hospital Hassan II, 30040 Fez, Morocco, Tel:

+212661670690, Email adil.mallouki@gmail.com

Received: July 31, 2018 | Published: November 19, 2018

\section{Introduction}

Traumatic lesions of the renal artery, classified as stage 5 according to the ASST classification (American Society of the Surgery of Trauma), are extremely rare and represent 1 to $4 \%$ of all the renal traumatisms. ${ }^{1}$ These lesions are most often encountered in polytrauma context; delayed diagnosis is due to the frequent absence of hematuria. For these severe traumas, the debate persists between supporters of a conservative attitude and those who advocate surgery. Technological advances in interventional radiology, endourology and the enhanced supportive measures in intensive care units allows adopting a conservative treatment even in the most severe trauma. ${ }^{2-6}$ The revascularization treatment is associated with high risks. Subsequently the conservative attitude seems to be a sure way to reduce the care-associated morbidity of those lesions based on enhanced supportive measures and regular monitoring. We report a case treated in our department of acute posttraumatic left renal ischemia where surveillance under improved supportive measures was adopted, on $18 \mathrm{YO}$ men, with intrest for all the specific characters of this pathology.

\section{Case report}

An 18-year-old patient, with no specific history, was admitted to the emergency department for treatment of polytrauma following a traffic accident. 3hours before admission, the patient who was the main driver, was ejected from a moped after being hit by a car. The patient was admitted immediately to the Crash room, with a GCS15 in a state of hemodynamic and respiratory instability, hypotension with $90 / 60 \mathrm{mmHg}$, no externalized hemorrhage, His initial hemoglobin was at $6 \mathrm{~g} / \mathrm{dL}$ with normal renal function. After enhanced resuscitation measures, the patient was hemodynamically and respiratory stabilized, and the initial founding was; a diffuse abdominal contraction, associated with left lower back pain and macroscopic hematuria.

Whole body CT Scanner was performed 5hours after the accident Figure 1, which revealed a laceration fracture of the spleen responsible for a low abundant intraperitoneal hematoma, associated with left renal ischemia due to a sub intimal damage on the left renal artery wall, with formation of a secondary obstructive thrombus, in addition to a left peri-renal hematoma of $16 \mathrm{~mm}$, without parenchymal lesions or extravasation of contrast, otherwise ; left vein, the right kidney and his pedicle were intact.

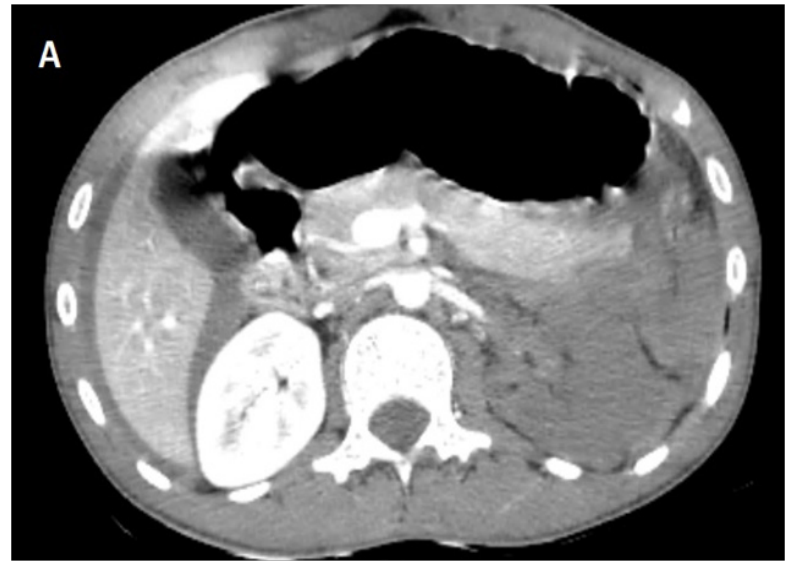

Figure I(A) Contrast enhanced CT abdominal scan.

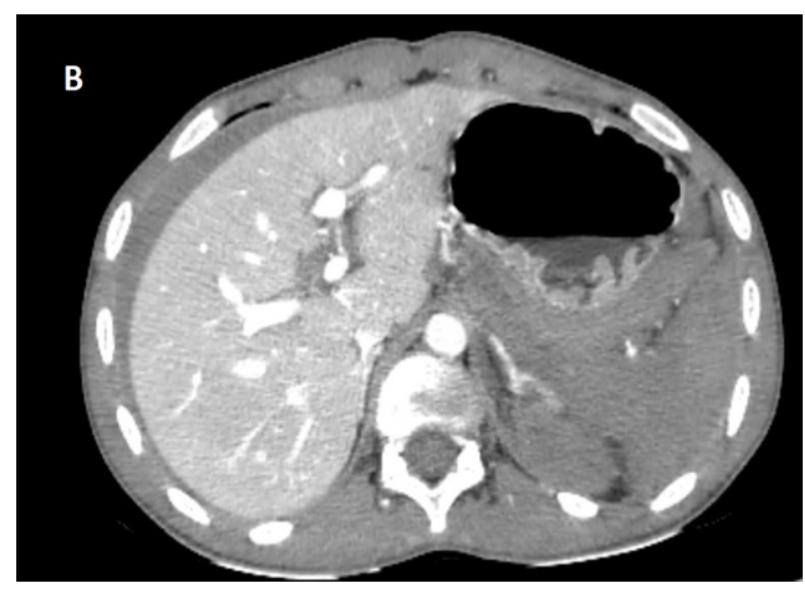

Figure I (B) Contrast enhanced CT abdominal scan. 


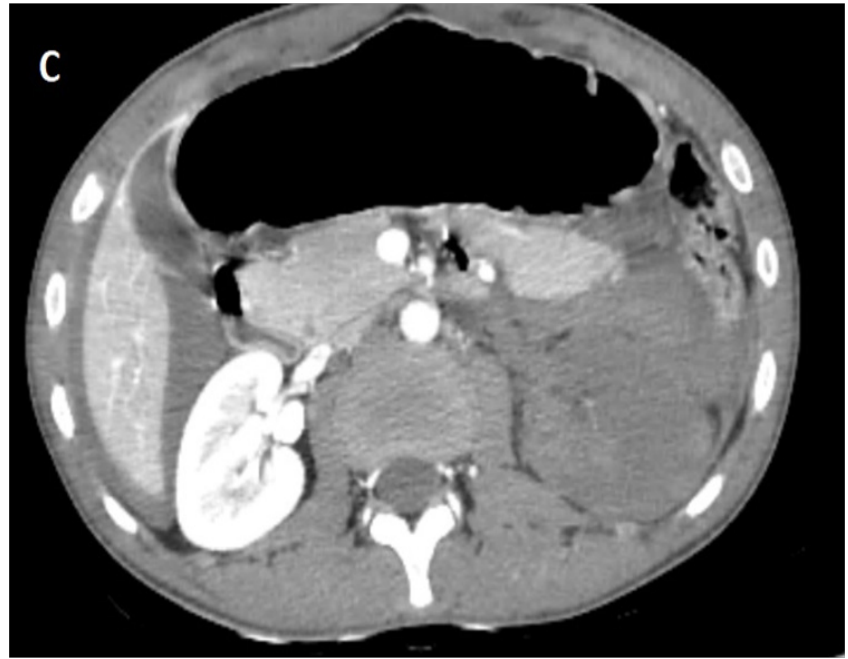

Figure I (C) Contrast enhanced CT abdominal scan.

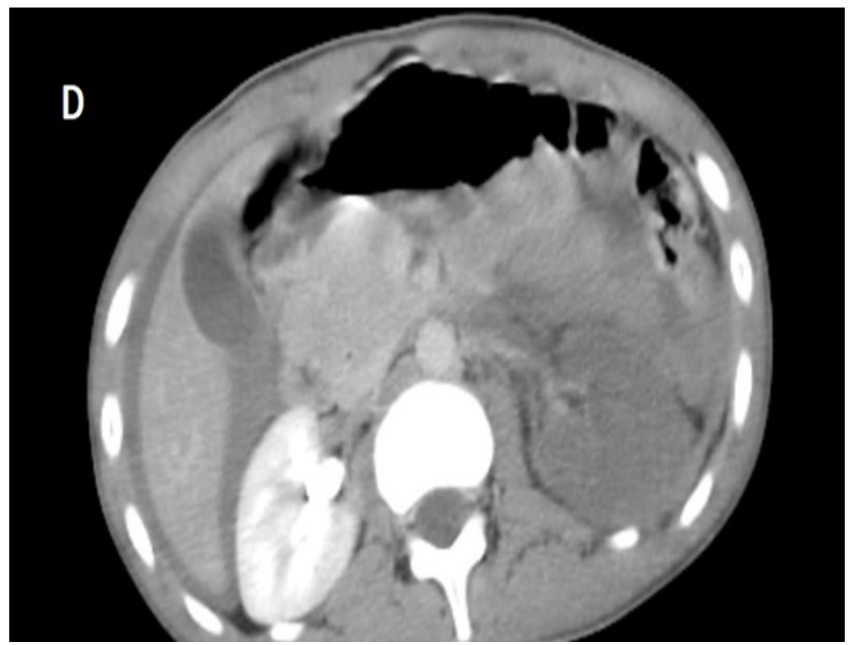

Figure I (D) Late acquisition of contrast enhanced CT abdominal scan.

laceration fracture of the spleen responsible for a low abundant intraperitoneal hematoma, associated with left renal ischemia due to a sub intimal damage on the left renal artery wall, with formation of a secondary obstructive thrombus, in addition to a left peri-renal hematoma of $16 \mathrm{~mm}$, without parenchymal lesions or extravasation of contrast, otherwise ; left vein, the right kidney and his pedicle were intact.

In the light of this radiological assessment, and after multidisciplinary concentration (vascular surgeons, urologists, radiologists and intensive care physicians), it was decided to adopt a conservative attitude with strict and regular monitoring, in an intensive care unit, with twice-daily hemogram. In order to detect any deglobulization, hypertension or infectious signs.

A Contrast enhanced computed tomography control was performed at $\mathrm{D}+7$ showing a sudden stop of the Contrast progression at the initial portion of the left renal artery, leading to total left renal ischemia, with stability of the perirenal hematoma, spleen injury and no extravasation at the late acquisitions.

The evolution during his stay in intensive care unit was favorable under a simple analgesic treatment, management of hypertension and a good rehydration, so the patient was transferred to visceral surgery department, with a follow-up program by imaging control in 3months and 6months.

\section{Discussion}

Injury of the renal pedicle is rare. They are frequently unilateral (left) and associated with polytrauma where intra-abdominal lesions predominates. ${ }^{1}$ Their proportion represents $2.5 \%$ to $4 \%$ of all the kidney injuries of patients admitted to surgical emergencies. ${ }^{3}$ Complete arterial thrombosis is even rarer. ${ }^{2,3}$ Thus, over a period of 15years Haas et al. described 12post-traumatic arterial obstructions, including only one case of bilateral obstruction. ${ }^{4}$ The validation of the ASST classification (American Society of the Surgery of Trauma) has lead to a better analysis and evidence based decision making of those lesions. ${ }^{2}$

Two main mechanisms explain the lesions observed during closed kidney trauma. The main mechanism is that of transmitting forces on the kidney surface that correspond to a lumbar or abdominal impact. The second mechanism is that of the antero-posterior or cephalocaudal movements of the kidney during sudden decelerations; the kidney being maintained only by its pedicle and the uretero-pelvic junction. This mechanism is more deleterious on the left side because of the absence of the liver, which plays the role of a support structure limiting the movements of the kidney and its pedicle.

The tension on the pedicle causes fracture of the most fragile tunic, the intima, which is responsible for the formation of a thrombus interrupting the arterial circulation at the origin of acute renal ischemia. ${ }^{8}$ Clinically in major renal traumas, lumbar pain is most often associated with gross hematuria or signs of shock in cases of complete pedicle rupture, with the formation of a retroperitoneal hematoma. ${ }^{2,3}$ The diagnosis is often delayed because of clinical symptoms; the absence of macroscopic or microscopic hematuria is noted in $18 \%$ to $36 \%$ of cases. $^{4}$

In our observation, the clinical feature was less obvious, the patient was admitted to emergency in a state of hemodynamic instability, associated with a diffuse abdominal defense, left lumbar back pain, and gross hematuria. Those clinical signs, suggesting the presence of severe renal trauma and probably other intra-abdominal lesions, led immediately to perform a radiological assessment after resuscitation measures.

The contrast enhanced computed tomography of the Abdomen (Angioscan) is the cornerstone asset in the study of kidney injuries, ${ }^{2,3}$ it help identify the associated lesions and staging renal injury. It allows, in one hand, to locate the parenchymal lesions, to seek an extravasation of the contrast, and to locate the avascular areas. And in the other hand it is used to quantify the importance of retroperitoneal hematoma, to check the status of the contralateral kidney (morphology and function) and to investigate the presence or absence of pedicular lesions and intraperitoneal organs. ${ }^{9}$ In addition to CT, new technologies including endo-urological drainage have emerged; post-traumatic resuscitation has improved considerably; Interventional radiology developed with new minimally invasive treatment techniques (embolizations, stent). ${ }^{9}$

The treatment of those pedicular lesions may involve: simple observation or surgery (total or partial nephrectomy, direct vascular repair, arterial bypass and auto-transplantation). The choice between one or the other of these methods is controversial and depends on the delay in diagnosis, the existence or not of associated lesions, the surgeon's experience, the type of lesion, the contralateral kidney status, and hemodynamics of the patient. ${ }^{8,9}$ 
The chances to successful surgical revascularization depend on one major element: the time to care; the delay of management, thus, the chances of success go from $80 \%$ to $57 \%$ from 12 hours to 18 hours. The importance of this delay, recognized by most authors, varies according to the teams: less than 5 hours ${ }^{4}$ and less than 12 hours. $^{2}$

In the light of evidence-based literature review, surgical revascularization for unilateral injury should be performed only if the patient is hemodynamically stable and the duration of ischemia is less than 5hours. ${ }^{4}$ If the duration of ischemia is greater than 5hours and the contralateral kidney has a normal function, surveillance seems to be a rational approach. If surgical exploration is required (ischemia greater than 5hours) for associated intra-abdominal injury, nephrectomy can be considered. ${ }^{4,9}$ Furthermore, revascularization is attempted regardless of the duration of ischemia if the lesion is bilateral, in front of a single kidney or grafted kidney.

However, complications following trauma should not be ignored, and are dependent on the lesion grade and the management method. ${ }^{2}$ Early complications occur in the weeks following the trauma, and its dominated by the resumption of bleeding, urinoma, and abscesses. Late complications after kidney injury include: hypertension, hydronephrosis, lithiasis, renal atrophy, renal failure, chronic pyelonephritis, and arteriovenous fistulas. Post-traumatic hypertension is caused by areas of renal ischemia that stimulates the renin-angiotensin aldosterone system. This complication is rare, it can occur several years after the trauma; and may be controlled by medical or surgical treatment. ${ }^{2}$

In the situation we reported, the decision-making of a conservative attitude was based on several features; delayed diagnosis (more than 5 hours after the trauma), left renal ischemia by unilateral complete thrombosis and the existence of retroperitoneal peri-renal hematoma with splenic laceration fracture, which contraindicates any anticoagulation after eventual surgical or endovascular surgery.

\section{Conclusion}

Post-traumatic renal ischemia is a rare representation of abdominal trauma, with low rates of renal function recovery, regardless of the therapeutic approach that has been considered. With the exception of single or bilateral renal lesions, or ischemia delay of less than 5hours, it does not seem desirable to attempt surgical revascularization. The management of left renal ischemia due to a sub intimal damage on the artery wall seems confusing, Consequently the conservative attitude seems to be a sure way to reduce the care-associated morbidity of those lesions based on enhanced supportive measures and regular follow-up especially long-term blood pressure monitoring.

\section{Acknowledgments}

None.

\section{Conflict of interests}

Authors declare that there is no conflict of interest

\section{References}

1. Zoulati M. Dissection traumatique de l'artère rénale gauche chez un adulte jeune. JMV-Journal de Médecine Vasculaire. 2018.

2. Abdelkader Saidi, Jean Luc Descotes, Christian Sengel, et al. Prise en charge des traumatismes fermés du rein. Progrès en Urologie. 2004; 14:461-471.

3. Jean-Alexandre long, Arnaud manel, sébastien penillon. Progrès en Urologie. 2004;14:302-309.

4. Haas CA, Dinchmank H, Nasrallahp F, et al. Traumatic renal artery occlusion: A 15-Year Review. J Urol. 1998;45(3):557-561.

5. Santuccir A, Mc Aninchj M. Grade 4 renal injuries: evaluation, treatment, and outcome. World J Surg. 2001;25:1565-1572.

6. Dinkel HS, Danuser H, TRILLER J. Blunt renal trauma: minimally invasive management with micro catheterembolization-experience in nine patients. Radiology. 2002;223:723-730.

7. Chatelain C. Essai de classification des lésions et proposition d'une tactique thérapeutique dans les traumatismes fermés récents du rein. Ann Urol.1981;15:210-214.

8. Merrot T, Portier F, Galinier P, et al. Traumatismes du pédicule rénal chez l'enfant. A propos de deux cas de revascularisation tardive par prothèse endovasculaire. Prog Urol. 2000;10:277-281.

9. Henry PC, Chabannese E, Bernardini S, et al. Prise en charge actuelle des traumatismes graves du rein. Prog Urol. 2002;12(4):579-586. 